ClinicAl Vistas

\section{A case of recurrent}

\section{meningitis}

A 20 year-old man presented to the emergency department with a 4-hour history of severe bifrontal headache, neck pain and vomiting associated with fever and a I-week history of productive cough. About 5 months earlier he had been admitted to another hospital with Streptococcus pneumoniae meningitis.

On presentation, the patient was found to have fever, tachycardia, nuchal rigidity, positive Kernig and Brudzinski signs and leukocytosis. Lumbar puncture revealed an elevated leukocyte count and neutrophilia and no detectable glucose, although Gram's staining of a sample of his cerebrospinal fluid (CSF) was negative. The patient's condition improved dramatically over 24 hours after empirical treatment with ceftriaxone, vancomycin and dexamethasone. Blood cultures eventually grew $S$. pneumoniae sensitive to penicillin.
The source of the patient's recurrent meningitis was unclear. His complement and immunoglobulin levels and the rest of his blood counts were normal. A head CT scan and chest radiograph appeared normal. Upon further questioning, the patient reported a persistent nasal discharge of clear fluid that had begun a year earlier following a motor vehicle collision, during which his airbag had failed to deploy and his face had struck the steering wheel. Other than pain and facial bruising, he had experienced no other obvious injuries and had not sought medical attention following the crash. His sense of smell was preserved bilaterally.

CT direct coronal imaging revealed a bony defect of the anterior cranial fossa on the left side that contained soft tissue, with associated remodelling of the crista galli and fovea ethmoidalis (Fig. I). These findings indicated a long-standing process that was probably congenital in nature. MRI coronal imaging, including fast spin echo (FSE) T2-weighted imaging (Fig. 2), demonstrated brain tissue within the defect extending into the nasal cavity. This finding suggested that the motor vehicle collision caused shearing of the overlying dural membrane to create a CSF fistula. The patient underwent elective bifrontal craniotomy, during which an encephalocele was found and repaired. His postoperative course was uncomplicated.

Recurrent bacterial meningitis is rare and should prompt a search for an underlying immunologic or anatomic cause. ${ }^{1-3}$ Immunologic abnormalities caused by complement or immunoglobulin deficiencies, asplenia or functional hyposplenis $\mathrm{m}^{2}$ are suggested by a history of recurrent infections of the ear, sinuses or respiratory tract and commonly involve encapsulated organisms such as $S$. pneumoniae, Neisseria meningitidis and Haemophilus influenzae. Structural defects usually present in infancy or childhood and include congenital causes such as congenital bony defects of the skull base or middle ear, meningoceles, encephaloceles, and cranio-

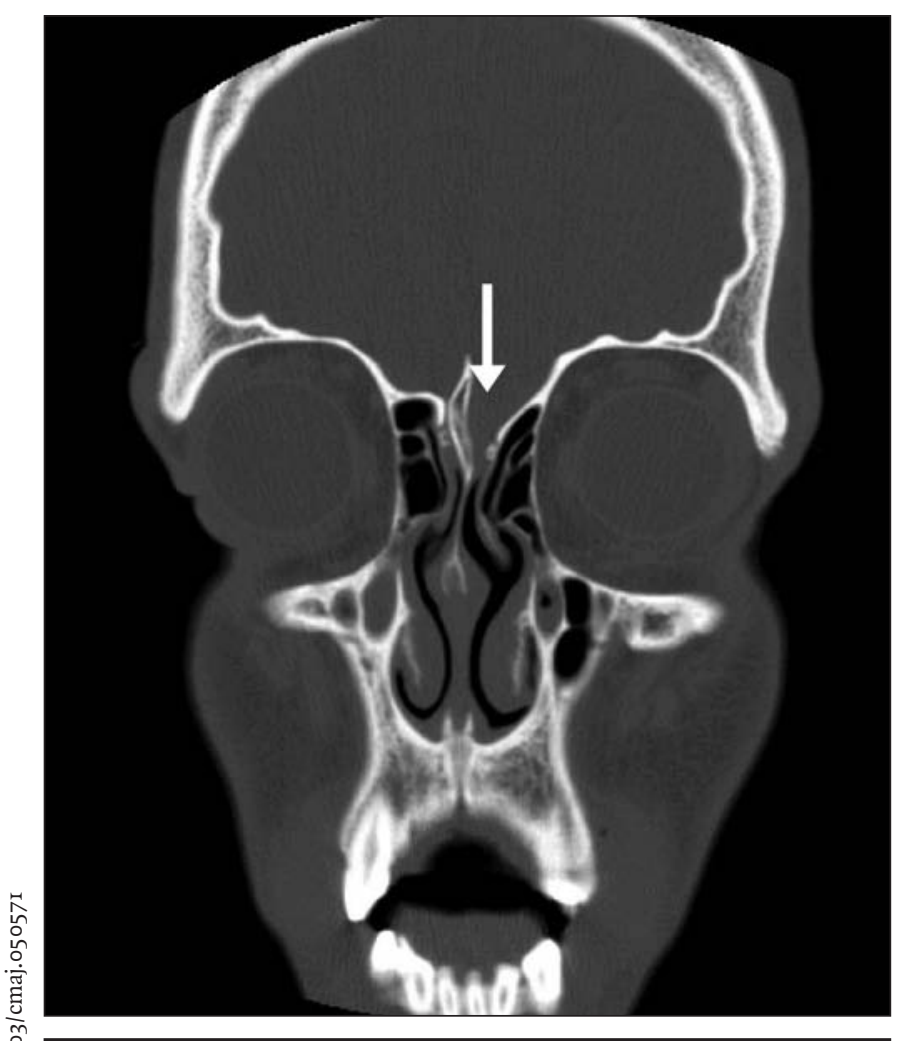

Fig. 1: CT direct coronal image demonstrating defect of the anterior cranial fossa on the left side (arrow).

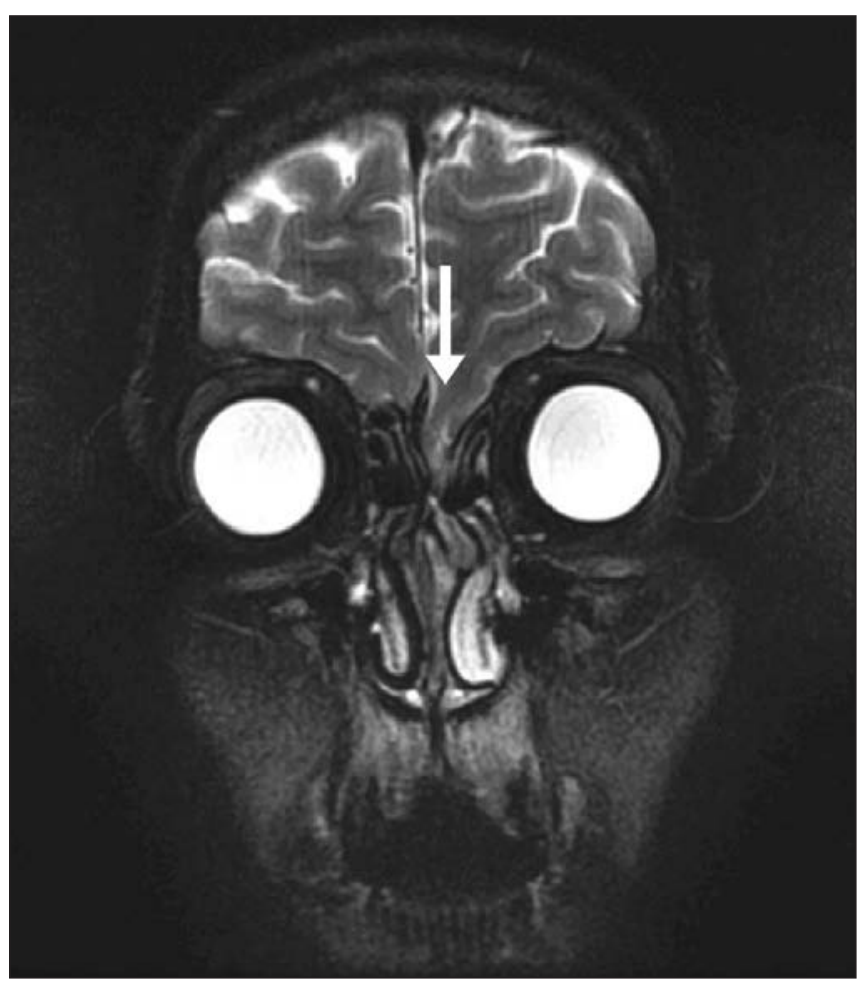

Fig. 2: Fast spin echo T2-weighted MRI coronal image demonstrating brain tissue extending into the left aspect of the nasal cavity (arrow). 
or spinal-dermal sinuses; acquired causes are usually related to skull fractures. ${ }^{2,4}$

Box 1: Features of meningitis resulting from basal skull defects

- Infection may occur long after the defect develops, which makes diagnosis and causality assessment more difficult

- Patients with cerebrospinal fluid rhinorrhea may misattribute the symptom to allergic rhinitis

- Sense of smell may be preserved if the fracture is distal to the olfactory bulb

- Routine axial imaging may not detect subtle abnormalities
The clinical course of our patient highlights 4 important features of meningitis resulting from basal skull defects (Box I). Direct thin-section CT coronal imaging, or thin-section axial imaging with reformatted coronal imaging, is the diagnostic test of choice for detecting such fractures. MRI, especially FSE T2-weighted coronal imaging, can reveal whether there is brain tissue, CSF or both within the defect. When anatomic defects are found, surgical repair is warranted to prevent recurrent episodes of meningitis.

\section{Erin B. Norris}

Department of Medicine

Perry Cooper

Department of Radiology

\section{David N. Juurlink}

Department of Medicine

University of Toronto

Sunnybrook and Women's College

Health Sciences Centre

Toronto, Ont.

This article has been peer reviewed.

Competing interests: None declared.

\section{REFERENCES}

I. Ginsberg L. Difficult and recurrent meningitis. J Neurol Neurosurg Psychiatry 2004;75(Suppl I):ir6-2I

2. Carrol ED, Latif AH, Misbah SA, et al. Recurrent bacterial meningitis: the need for sensitive imaging. BMJ 200I;323:50I-3.

3. Ford $\mathrm{H}$, Wright J. Recurrent bacterial meningitis in adults: a case series. JInfect I996;33:I3I-3.

4. Hosoglu S, Ayaz C, Ceviz A, et al. Recurrent bacterial meningitis: a 6-year experience in adult patients. J Infect I $997 ; 35: 55-62$.

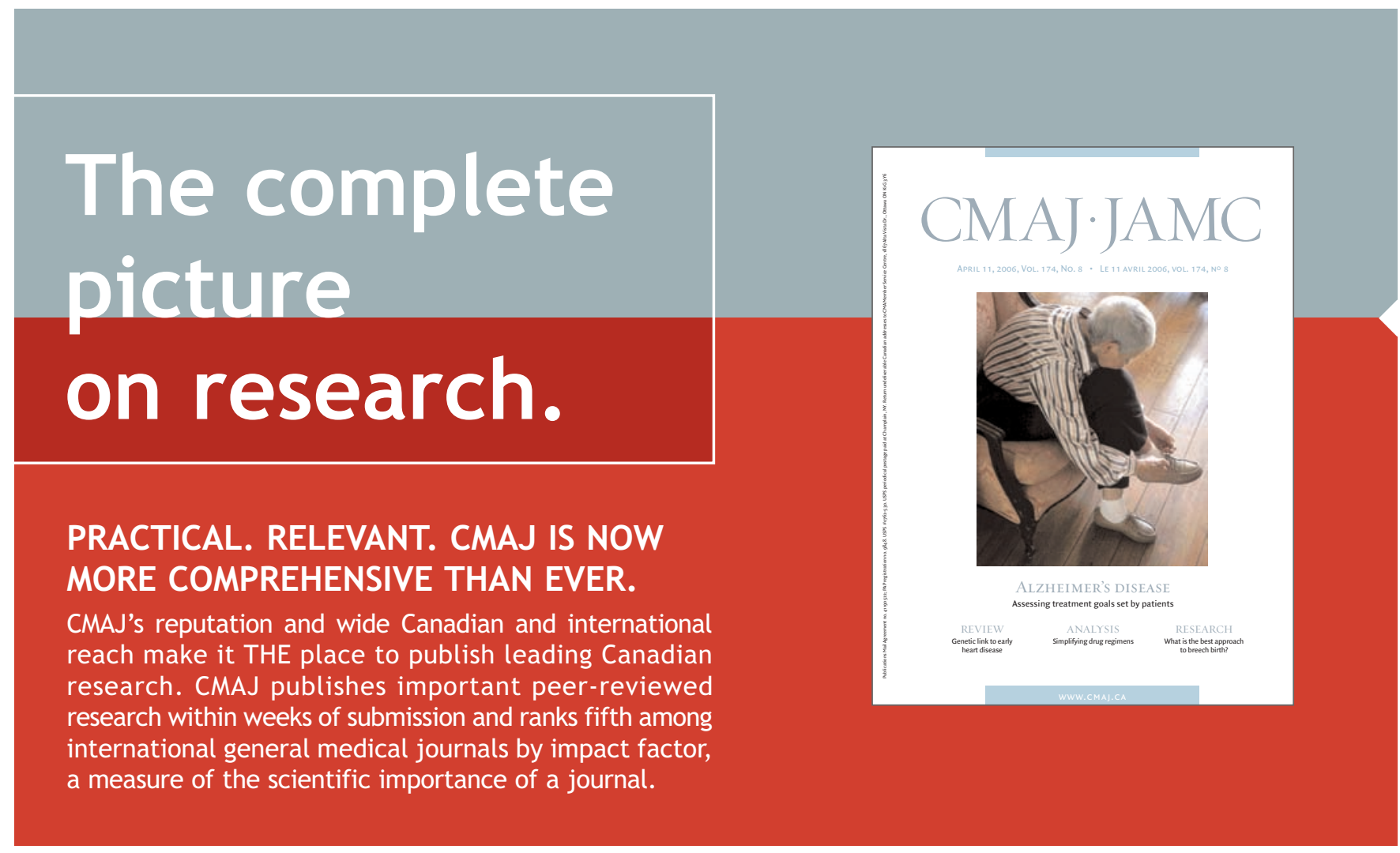

The essential read. ${ }^{\mathrm{TM}}$ 